\title{
A Customizable Game-inspired Application for Memory Stimulation
}

\author{
Rodrigo Rocha ${ }^{1}$, Davide Carneiro ${ }^{1,3}$, Ana P. Pinheiro ${ }^{2}$, and Paulo Novais ${ }^{3}$ \\ 1 CIICESI, ESTG, Polytechnic Institute of Porto \\ Felgueiras, Portugal \\ \{8140411, dcarneiro\}@estg.ipp.pt \\ 2 Faculdade de Psicologia, Universidade de Lisboa, Lisbon, Portugal \\ appinheiro@psicologia.ulisboa.pt \\ 3 Algoritmi Centre/Department of Informatics, Universidade do Minho, Portugal \\ pjon@di.uminho.pt
}

\begin{abstract}
Demographic changes are leading to a growing older population (>65 years), with repercussions on age-related conditions. From a Computer Science perspective, this also means that there will soon be a significant number of users with changes in perceptual and motor skill capacities. The goal of this work is to develop an environment to support the preservation of memory and functional capacities of the elderly. Health professionals will be able to set up and personalize immersive and realistic scenarios with high ecological validity composed of visual, auditory, and physical stimuli. Patients will navigate through and interact with these scenarios and stimulate memory functions by later recalling distinct aspects of the different exercises of the tool. The long-term goal is to build a behavioral model of how older users interact with technology.
\end{abstract}

Keywords: Real-Time Analytics, Big Data, NoSQL Databases, Behavioral Biometrics

\section{Introduction}

Demographic changes are leading to a growing population of older people, with repercussions on age-related conditions such as Mild Cognitive Impairment (MCI) $[1,2]$. Pathological aging is related to a noticeable and measurable decline in cognitive abilities, including memory, as well as changes in perceptual and motor skill capacities, considering what would be expected based on age and educational level. Among other consequences, these changes bring important implications for the design of ICT.

The primary aim of this multidisciplinary work is to develop an environment to stimulate and support the preservation of memory and functional capabilities of the elderly, mitigating or delaying negative consequences of ageing and promoting a more active and autonomous ageing process. Indeed, recent results show that memory remains plastic even in an older age: after mnemonic training, the average elderly person can be expected to perform at the $77^{t h}$ percentile 
of the performance distribution of their age group $[3,4]$. However, the plasticity associated with mnemonic training appears to be largely specific to that training as improvement was found to be higher on tasks allowing for the use of the newly acquired mnemonic than on tasks not allowing for that use [5,6]. Similarly, [7] shows that although the methods used by top "mental athletes" allow them to memorize surprising amounts of information, the acquired abilities are rather irrelevant when performing daily activities. The main drawback in existing approaches is thus that most of them are based on mnemonics or similar mental tasks, that are hard to translate to skills needed in real-life $[8,9]$.

The development of a software environment for such a specific population also constitutes an unprecedented opportunity. Indeed, a population of older technology users will be a reality in a few decades. In this regard, one of the key issues of HCI designs is to understand the impact of older users' abilities and restrictions [5]. However, the inclusion of older people within the design cycle for information technology is until now limited to aspects such as usability or the graphical aspects of user interface.

The long-term goal of this work is thus to build a behavioral model of how old users interact with technological devices. This model will further our understanding of the specific interaction patterns of the elderly, supporting the development of more accessible and inclusive applications and devices. Moreover, it may unveil differences between healthy people and people with different profiles of cognitive impairment, with the potential of acting as an early diagnosis mechanism for age-related conditions such as MCI or dementia.

\section{Conceptualization}

In the proposed approach, the clinician is able to set up and personalize immersive and realistic scenarios, that will afterwards be navigated by the patients. Each scenario is composed of one or more rooms. Each room is composed of one or more still photos. Each photo may have other superimposed photos in specific coordinates and with a specific size (Figure 1, left). Each photo may also have one or more auditory stimuli associated, with specific playing patterns, which will be played when the patient visualizes the current photo. Each photo also has information describing its position relatively to other photos, which makes the scenarios navigable. Finally, each photo may also have "passages" to other rooms (Figure 1, right).

These navigable scenarios may portrait the home of the participant, a grocery store, or virtually any intended environment. Moreover, scenarios can be enriched and fully personalized with additional stimuli (e.g. family pictures, objects, sounds) to stimulate a specific cognitive ability or to recall specific types of memories (e.g. family members).

This is, in fact, one of the most promising features of this project. Recent studies show that the plasticity associated with mnemonic training (which is the most common approach to memory stimulation) appears to be largely specific to that training as improvement was found to be higher on tasks allowing for 

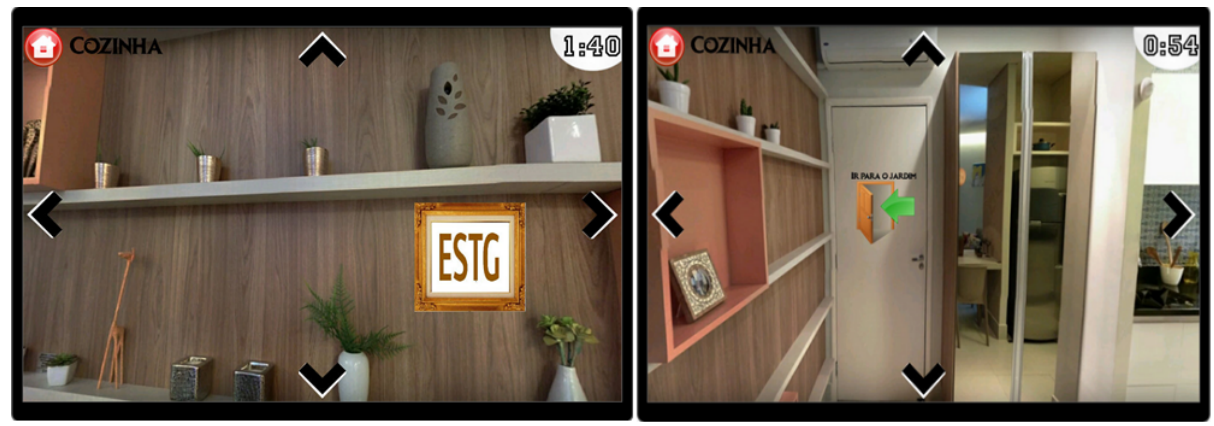

Fig. 1. Two still photos of one room of a given scenario. A custom picture has been superimposed by the clinician (the ESTG frame) in the left photo. The photo on the right shows a passage to another room (garden).

the use of the newly acquired mnemonic $[3,10]$. We believe that if clinicians design realistic scenarios, with realistic stimuli, this will allow patients to reuse the cognitive improvements acquired while using this application in their daily activities (e.g. go shopping, spend time with family members).

Patients use a mobile application to navigate through these scenarios ("Point and Click" style), when and as defined by the researcher. Later, patients recall their experience through specific exercises. These recall tasks may be of four different types: multiple choice (text and/or images), multiple choice single answer (text and/or images), numeric answer or free text.

Moreover, each recall task may reference any of the specific auditory or visual stimuli present in the corresponding scenario (e.g. "In which room was this image located?"). During these recall tasks, memory performance is automatically measured and quantified, providing valuable feedback to the researcher through real-time analytics.

\section{Architecture}

The architecture that implements the proposed system is composed of three main elements: a mobile application, the server, and tools for administration and realtime analytics (Figure 2). There are also two different types of users: patients, who interact with the mobile application, and clinicians, who interact with the analytics and administration system. Each of these elements is described in the following sub-sections.

\subsection{Server}

The server is the central element of this architecture. It is composed of three main components: a NoSQL database, a node.js server, and a Spark instance.

All the main functionalities of the server are implemented by node.js. This includes services for uploading media to the server (e.g. sounds, images) that 


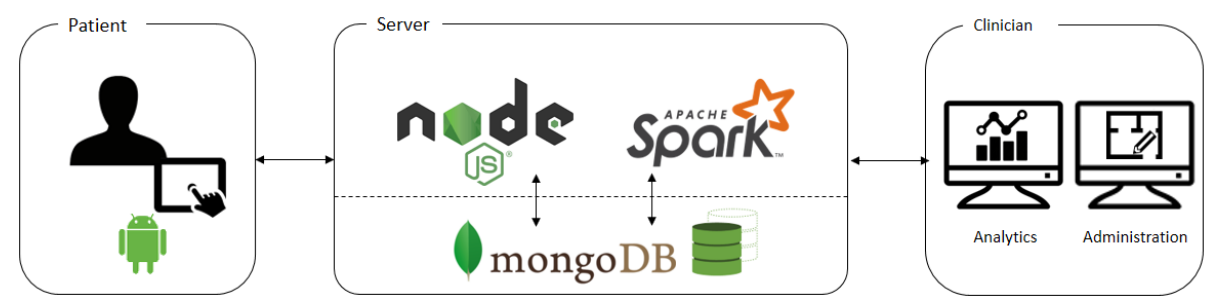

Fig. 2. Architecture of the developed system.

can later be used to construct scenarios, mediating access to the database, and a HTTP web server for communicating with external applications.

The NoSQL database stores all the relevant data, which includes:

- User Data - Identifying and authentication data. For patients this also includes socio-demographic variables;

- Scenarios - This collection contains the structural information of each scenario, including rooms/photos, relative positions, passages, superimposed photos and auditory stimuli;

- Recall Tasks - Collection of all recall tasks created by the clinicians, with information describing type of task, possible answers (in case of multiple choice), correct answer, referenced stimuli (if any) and target patient/scenario;

- Results - Contains all the data collected to measure the patients' performance during recall tasks;

- Behavioural Data - Describes all the data collected from the patients' interaction with the mobile devices, such as touch patterns and time between decisions;

- Administration - Contains data associating scenarios, to patients, to recall tasks. That is, which scenarios/recall tasks should be made available to which patients, at which moment and during how much time.

Finally, the Spark instance is used for data processing and machine learning tasks, namely for computing measures of cognitive performance and for analyzing patients' interaction patterns.

\subsection{Mobile Application}

The mobile application is developed for Android tablets. It is the patients' point of interaction with the system. It is through this application that the patient navigates in the scenarios that are appointed by the clinician. It is also through this application that the patient participates in the recall tasks.

The application checks, at regular intervals, if there are available scenarios/recall tasks for the logged in patient. Where there are, the application downloads the corresponding media (photos and sounds) as well as the meta-data that describes how photos and sounds should be displayed. When the download is complete, the scenario/recall task becomes available to the patient. 
The application also collects two types of data transparently (Section 4). This data is temporarily stored locally in the device, and sent to the server when a data connection is available.

\subsection{Analytics and Administration}

The analytics and administration component implements functionalities targeted at the clinician. The Analytics module provides statistic measures about the different variables collected, describing memory performance and interaction patterns over time, for each user. These variables are very important for clinicians to monitor and evaluate the evolution of the patients' behaviour and cognitive performance over time.

The Administration module is designed for clinicians to design scenarios and recall asks. Clinicians start by uploading the necessary media (e.g. photos and sounds) that they will use. Then, they design the scenario by creating rooms, assigning photos to rooms, placing them relatively to each other, superimposing other photos/sounds (if desired) and specifying passages. Finally, through this module the clinicians can also assign scenarios/recall tasks to specific patients, as well as set the period of availability.

\section{Features}

The mobile application collects data that are used to produce two different types of features. Operational features describe what the patient does in the application, while behavioral features describe how the patient does it.

Operational features are extracted from the patients actions while navigating through the scenarios and/or while carrying out recall tasks. The following features are available for the clinicians, all of which can be aggregated by patient/scenario/gender/age group/recall task or any combination of these attributes, allowing different views on the data:

- Correct answers - This is the main feature in assessing the cognitive performance of the patient. It can be provided in percentage or in absolute terms;

- Time between decisions - Quantifies the time, in milliseconds, between each two consecutive decisions of the patient;

- Actions log - This feature quantifies the events and duration of each individual activity of a given participant (e.g. visiting a room, completing a recall task, starting/ending a specific question of a recall task).

These features allow, above all, clinicians to evaluate the cognitive performance of patients with different granularities (e.g. over a long period of time, in a specific recall task). This analysis can also be performed by group, gender, age group, among others. The three features provide significant information for quantifying cognitive performance. The quantification of correct answers is a direct measure. The time between decisions quantifies, in a way, the fitness of this 
performance, i.e., the faster the decisions the fitter (in principle) the brain is. Finally, the action has similar uses as it quantifies how much time each patient spent in each question/task/room, with longer times being associated to less cognitive performance.

On the other hand, there are the behavioural features, which describe mostly the interaction of the patient with the device. These include:

- Touch duration - The duration of each individual touch on the screen;

- Touch intensity - The average, minimum and maximum values of the intensity exerted by the finger on the screen, for each touch;

- Touch area - The average, minimum and maximum values of the area occupied by the finger on the screen, for each touch;

- Intensity values - The sequence of values of intensity generated during each touch, from the moment the finger first touches the screen to the moment it is lifted;

- Area values - The different areas of finger in contact with the screen, in each touch, from the moment the finger first touches the screen to the moment it is lifted;

- Type of action - The actions on the screen can be further characterized (e.g. touch on an active control vs. touch on a layout inactive element).

These features describe how the patient is interacting with the device. They provide new types of information previously not considered in this kind of applications, as explored in Section 5. Indeed, and as put forward in the introductory section, we believe that in the long term and after the collection of extensive interaction data from different groups of patients, we will be able to find differences in interaction patterns due to socio-demographic variables such as gender, age, occupation or health conditions.

\section{Validation}

This section describes some of the data collected with this application, from patients with different characteristics. Specifically, and to highlight the potential interest of this approach in terms of Human-Computer Interaction, in this section we analyze data collected from four different users: two male (one young and one older) and two female (one young and one older). This is not meant to be a representative data analysis but only the validation of the proposed hypothesis, that is, showing that age- and gender-based differences may exist. We are now collecting and analyzing data from a large group of users, in order to study these hypothesis. Given the scope of the paper, in this section we focus on the behavioral features described in Section 4.

Figure 3 highlights the differences according to gender and age of two of the variables: touch duration and touch intensity. It shows that older people tend to have longer and also stronger touches on the touch screen, with differences being more evident in duration. These results are further detailed in Table 1 

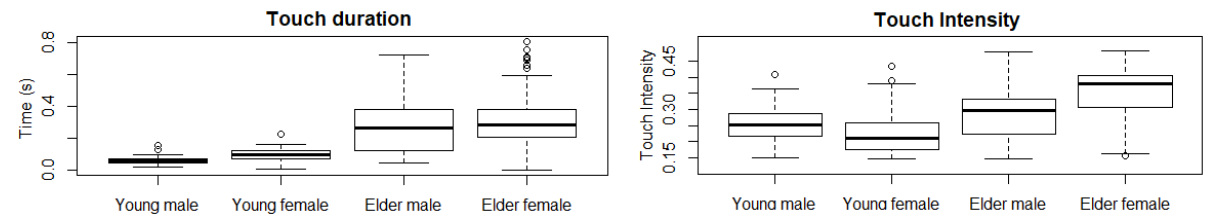

Fig. 3. Distribution of the time between decisions of four different patients: older patients tend to take longer to make decisions when visiting the scenarios or completing recall tasks.

Table 1. General statistics of touch duration and intensity for both genders and age groups.

\begin{tabular}{lcccc} 
& \multicolumn{4}{c}{ Touch Duration Touch Intensity } \\
\hline & $\bar{x}$ & $\sigma$ & $\bar{x}$ & $\sigma$ \\
\hline Young Male & 0.061 & 0.022 & 0.255 & 0.052 \\
Young Female & 0.097 & 0.041 & 0.228 & 0.069 \\
Elder Male & 0.279 & 0.174 & 0.285 & 0.082 \\
Elder Female & 0.315 & 0.178 & 0.354 & 0.080 \\
\hline
\end{tabular}

Another interesting feature that can be built from the collected data is the patient's touch pattern, i.e., how touch intensity varies over time during the touch. In this approach, each patient's touch pattern is modelled by fitting a quadratic function to the data. On the one hand, this provides a combined analysis of these two factors. Moreover, the coefficients of the resulting quadratic function can be efficiently used to characterize the "general shape" of a patient's touch. We expect that this kind of data may allow for interesting applications such as user identification. Figure 4 shows the touch patterns of the four different patients, depicted in terms of the intensity values over time and the resulting quadratic function that models it: (a) young male, (b) elder male, (c) young female and (d) elder female.

\section{Conclusions}

In this paper we presented a game-inspired mobile application for stimulating memory, especially designed for older people or for people with cognitive disabilities. It has several key innovative aspects. First, it is a tool that can be completely personalized by the clinician, with each patient's specific needs in mind. It allows for the distributed and remote management and administration of interventions, facilitating the whole process. Finally, it acquires, in real-time, a wide range of data describing the patient's memory performance and interaction patterns. In this sense, it provides many new features that were, until now, not considered in this type of intervention. It can thus significantly contribute not only for the study of memory and its improvement but also for a better 

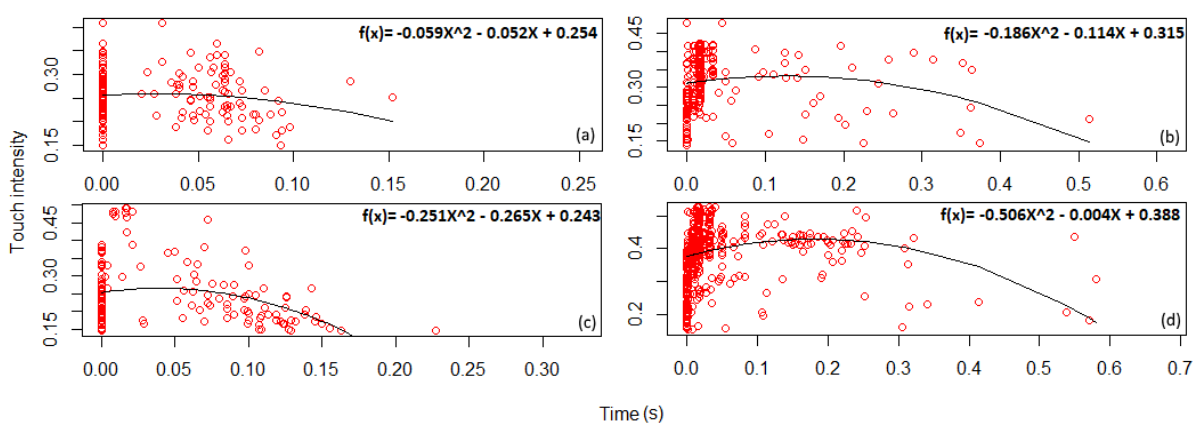

Fig. 4. Touch patterns (intensity over time) of the four patients: (a) young male, (b) elder male, (c) young female and (d) elder female.

understanding of Human-computer Interaction, especially in these slices of the population.

\section{References}

1. Petersen, R.C., Caracciolo, B., Brayne, C., Gauthier, S., Jelic, V., Fratiglioni, L.: Mild cognitive impairment: a concept in evolution. Journal of internal medicine 275(3) (2014) 214-228

2. DeSA, U.: World population prospects: the 2012 revision. Population division of the department of economic and social affairs of the United Nations Secretariat, New York (2013)

3. Verhaeghen, P., Marcoen, A., Goossens, L.: Improving memory performance in the aged through mnemonic training: a meta-analytic study. (1992)

4. Park, D.C., Bischof, G.N.: The aging mind: neuroplasticity in response to cognitive training. Dialogues in clinical neuroscience 15(1) (2013) 109

5. Jochems, N.: Designing tablet computers for the elderly a user-centered design approach. In: International Conference on Human Aspects of IT for the Aged Population, Springer (2016) 42-51

6. Verhaeghen, P.: Memory training and mnemonics. The Encyclopedia of Adulthood and Aging (2016)

7. Foer, J.: Moonwalking with Einstein: The art and science of remembering everything. Penguin (2012)

8. Pereira, G., Ninaus, M., Prada, R., Wood, G., Neuper, C., Paiva, A.: Free your brain a working memory training game. In: International Conference on Games and Learning Alliance, Springer (2014) 132-141

9. Jensen, K., Valente, A.: Development of a memory training game. In: Technologies of Inclusive Well-Being. Springer (2014) 25-38

10. Deveau, J., Jaeggi, S.M., Zordan, V., Phung, C., Seitz, A.R.: How to build better memory training games. Frontiers in systems neuroscience 8 (2015) 243 